\title{
Synchronization in the presence of time delays and inertia: Stability criteria
}

\author{
Dimitrios Prousalis $\odot^{*}$ and Lucas Wetzel $\odot^{\dagger}$ \\ Max Planck Institute for the Physics of Complex Systems, Dresden, Germany
}

(Received 19 July 2021; accepted 27 December 2021; published 18 January 2022)

\begin{abstract}
Linear stability of synchronized states in networks of delay-coupled oscillators depends on the type of interaction, the network, and oscillator properties. For inert oscillator response, found ubiquitously from biology to engineering, states with time-dependent frequencies can arise. These generate side bands in the frequency spectrum or lead to chaotic dynamics. The time delay introduces multistability of synchronized states and an exponential term in the characteristic equation. Stability analysis using the resulting transcendental characteristic equation is a difficult task and is usually carried out numerically. We derive criteria and conditions that enable fast and robust analytical linear stability analysis based on the system parameters. These apply to arbitrary network topologies, identical oscillators, and delays.
\end{abstract}

DOI: 10.1103/PhysRevE.105.014210

\section{INTRODUCTION}

Self-organized synchronization can be observed in chemical oscillators, embryonic development, circadian clocks, ranging to power grids and the orchestration of mobile communications and microelectronic and mechanical systems [1-10]. This type of synchronization has been considered for electronic networks since the 1980s due to its robustness and as its properties scale advantageously with growing system size $[11,12]$. In application, however, it did not prevail over hierarchical synchronization as the necessary theoretical framework to guide architecture design was not available [11]. Within phase oscillator models the dynamics in networks of coupled oscillators can be studied [13,14]. This includes the effects of inevitable time delays in the coupling. These lead to phenomena like multistability of synchronized states [15]. Another aspect of the oscillators' dynamics has recently come into focus, inert response to external stimuli. Examples are the inertia of mechanical oscillators, signal filtering in electronics, or biochemical transport and conversion processes in cellular oscillators $[16,17]$. Inert system response in secondorder phase models can trigger bifurcations of synchronized states with constant frequency $[18,19]$. Frequency modulation occurs, side bands arise in the spectrum, and synchronized states with constant phase relations become unstable. There are numerical tools that allow us to study such bifurcations [20]. Furthermore, the stability of synchronized states in networks of identical oscillators can be studied using, e.g., the

\footnotetext{
*dprou@pks.mpg.de

†lwetzel@pks.mpg.de
}

Published by the American Physical Society under the terms of the Creative Commons Attribution 4.0 International license. Further distribution of this work must maintain attribution to the author(s) and the published article's title, journal citation, and DOI. Open access publication funded by the Max Planck Society.
Master Stability Formalism [21]. This method extends the linear stability analysis in dynamical systems theory and introduces a so-called master stability function. Several works have built on that and studied specific systems with time-delayed coupling [22-27]. For example, in networks with inertia and processing delays, necessary and sufficient conditions for the asymptotic stability have been derived [28].

In this work we derive stability criteria for in- and antiphase synchronized states in networks of coupled oscillators with inertia and in the presence of transmission time delays. The criteria depend only on the physical properties of the oscillators and the network and can guide, e.g., the architecture design of synchronization layers in networks of mutually coupled electronic oscillators. We then use the criteria to obtain parameter space plots and discuss the linear stability of in- and antiphase synchronized states in general. Our criteria simplify studying the physical properties of synchronization over large parameter regimes and, e.g., large but finite time delays and large number of oscillators. We discuss how linear stability depends on physical properties such as time delay, inertia, damping or dissipation, interaction strength, and network topology. These generic concepts can then be related to application specific concepts like, e.g., the loop gain and bandwidth of electronic oscillators or the dissipation coefficients in power grids [29-32]. Additionally, we present a condition connecting these quantities. If fulfilled, linear stability is guaranteed, and hence no bifurcations occur.

\section{NETWORKS OF DELAY-COUPLED OSCILLATORS WITH INERTIA}

The dynamics in such networks can be studied within the following set of coupled delay-differential equations:

$$
m \ddot{\theta}_{k}(t)+\gamma \dot{\theta}_{k}(t)=\omega+\frac{K}{n_{k}} \sum_{l=1}^{N} c_{k l} h\left(\frac{\Delta \theta_{k l}(t, \tau)}{v}\right),
$$


where $k=1, \ldots, N$ indexes the $N$ oscillators in the network, $\omega \in \mathbb{R}$ denotes the intrinsic frequency, $h(\cdot)$ a periodic coupling function, $K \geqslant 0 \in \mathbb{R}$ the coupling strength, $m \geqslant 0 \in$ $\mathbb{R}$ an inertial parameter, $\gamma>0 \in \mathbb{R}$ a damping parameter, $n_{k} \geqslant 0 \in \mathbb{N}_{0}$ the number of inputs of oscillator $k, \theta_{i}(t) \in S^{1}$ for $i=\{k, l\}$ the phases of the oscillators' output signals with $\dot{\theta}$ and $\ddot{\theta}$ denoting their first and second time derivatives, and $c_{k l}$ the components of the network's adjacency matrix, being either 1 if there is a connection from oscillator $l$ to $k$ or 0 otherwise. $\Delta \theta_{k l}(t, \tau)=\theta_{l}(t-\tau)-\theta_{k}(t)$ is the phase difference between $k$ and an input $l$. Here $\tau \in \mathbb{R}$ denotes the cross-coupling time delay. $v>0 \in \mathbb{R}$ denotes the division of the instantaneous output frequency of the oscillators, e.g., induced by a frequency divider. This is well known from, e.g., periodic cross-coupling signals in networks of electronic oscillators [33]. Note that Eq. (1) reduces to the classical firstorder Kuramoto model for sinusoidal coupling $h(\cdot)=\sin (\cdot)$, zero coupling delay $\tau=0$, damping coefficient $\gamma=1$, and inertia $m=0$. We study in- and antiphase synchronized states and their linear stability making the ansatz

$$
\theta_{k}(t)=\Omega t+\beta_{k}+\epsilon q_{k}(t),
$$

where $\Omega$ denotes the frequency of a synchronized state, $\epsilon q_{k}(t)$ a small perturbation $(\epsilon \ll 1)$, and $\beta_{k}$ a phase offset. Using the ansatz (2) in Eq. (1) and Taylor expansion of $h(\cdot)$ with respect to $\epsilon$ we obtain the properties of synchronized states from $O\left(\epsilon^{0}\right)$

$$
\gamma \Omega=\omega+K h\left(-\frac{\Omega \tau+\beta}{v}\right),
$$

where $\beta / v=\left(\beta_{k}-\beta_{l}\right) / v$ equals 0 or $\pi$. This implies that inand antiphase synchronized states self-organize at the level of the divided cross-coupling signals. As a consequence, the phase relations with respect to the output frequency $\Omega$ can be in antiphase only if $v$ is odd since $\beta=\{0, v \pi\}$. The dynamics of the perturbations $q_{k}(t)$ are given by $O\left(\epsilon^{1}\right)$ of the Taylor expansion

$$
m \ddot{q}_{k}(t)+\gamma \dot{q}_{k}(t)=\frac{\alpha}{n_{k}} \sum_{l=1}^{N} c_{k l}\left[q_{l}(t-\tau)-q_{k}(t)\right],
$$

where $\alpha=\frac{K}{v} h^{\prime}\left(\frac{-\Omega \tau+\beta}{v}\right)$ denotes a steady-state parameter, characterized by $\{\Omega, \beta\}$. Substituting $q_{k}(t)=q_{k}^{0} e^{\lambda t}$ into Eq. (4), the dynamics of small perturbations in the Laplace domain are obtained as

$$
e^{\lambda \tau}\left(m \lambda^{2}+\gamma \lambda+\alpha\right) q_{k}^{0}=\alpha \sum_{l=1}^{N} d_{k l} q_{l}^{0},
$$

where $d_{k l}=c_{k l} / n_{k}$ are the components of the normalized adjacency matrix $\mathbb{D}$, and $\lambda=\sigma+i \mu$ the complex frequency. Rewriting in matrix form, we identify the eigenvalue problem $\zeta \vec{q}=\mathbb{D} \vec{q}$ and the characteristic equation

$$
\lambda^{2}+\omega_{c} \gamma \lambda+\alpha \omega_{c}\left(1-\zeta e^{-\lambda \tau}\right)=0,
$$

where $\omega_{c}=m^{-1}$ and $\zeta=|\zeta| e^{i \Psi}$ are the eigenvalues of the normalized adjacency matrix $\mathbb{D}[34]$. These $\zeta$ relate to the perturbation modes in the network, and each generates an infinite discrete set $\Lambda_{\zeta}$ of solutions $\lambda$. For diagonalizable $\mathbb{D}$ arbitrary perturbations can be expressed by linear combinations of $\vec{q}$, the eigenvectors. The eigenvector that induces a global shift of all phases $\vec{q}=(1,1, \ldots, 1)$ has the eigenvalue $\zeta=1$ since $\sum_{l} d_{k l}=1$. It does not affect the synchrony of the system and will be excluded in the following discussions. From dynamical systems theory it is known that the largest $\sigma$ in the union $\cup_{\zeta \neq 1} \Lambda_{\zeta}$ dominates the long-term dynamics of the perturbations. If the largest $\sigma>0$, then perturbations grow, and the state is linearly unstable. If all $\sigma<0$ the system is linearly stable. From Eq. (6) we find that if $\alpha=0$, then $\lambda_{1}=0$ and $\lambda_{2}=-\omega_{c} \gamma$. Hence, $\alpha=0$ relates to marginally stable solutions and will not be considered in the following.

\section{DERIVATION OF STABILITY CRITERIA}

For first-order Kuramoto models with time delays, i.e., without inertia, Earl and Strogatz derived a criterion that determines linear stability of synchronized states [35]. It concludes that synchronized states in networks of delay-coupled oscillators with arbitrary coupling topology are linearly stable if and only if $\alpha=K h^{\prime}(-\Omega \tau)>0$. For Kuramoto models with time delay and inertia it has been shown that this criterion cannot sufficiently predict linear stability [34]. There is no known closed form solution to second-order exponential polynomials like Eq. (6). Such solutions can be obtained numerically but require a careful choice of initial conditions and become increasingly difficult for large time delay and network size. In previous works, conditions that connect inertial properties with the interaction strengths and properties of the synchronized states that prevent instability have been found [11,30,36].

Here we introduce stability criteria that allow us to predict linear stability of in- and antiphase synchronized states in networks of delay-coupled oscillators with inertia for any set of parameters. Furthermore, we extend the previously found conditions and connect them to properties of the topology [30]. With $\lambda=\sigma+i \mu$ in Eq. (6) and separate the real and imaginary parts:

$$
\begin{gathered}
\sigma^{2}+\omega_{c} \gamma \sigma=-\alpha \omega_{c}\left[1-|\zeta| \cos (\mu \tau-\Psi) e^{-\sigma \tau}\right]+\mu^{2} \\
2 \sigma \mu=-\omega_{c}\left[\mu \gamma+\alpha|\zeta| \sin (\mu \tau-\Psi) e^{-\sigma \tau}\right]
\end{gathered}
$$

Squaring and adding these equations we obtain

$$
\begin{aligned}
& \left(\sigma^{2}-\mu^{2}+\omega_{c} \gamma \sigma+\alpha \omega_{c}\right)^{2}+\left(2 \sigma \mu+\omega_{c} \gamma \mu\right)^{2} \\
& \quad=\left(\alpha \omega_{c}|\zeta|\right)^{2} e^{-2 \sigma \tau} .
\end{aligned}
$$

We begin by addressing one direction of the known stability criterion presented in [35]. For second-order phase models we show that if $\alpha<0$, there always exists at least one $\sigma>0$, and hence the states in Eq. (3) are unstable. Setting $\alpha=-|\alpha|$ and $\rho=|\zeta| \cos (\mu \tau-\Psi)$ in Eq. (7a) we find after rearranging

$$
\sigma^{2}+\omega_{c} \gamma \sigma=|\alpha| \omega_{c}\left(1-\rho e^{-\sigma \tau}\right)+\mu^{2},
$$

where $\rho \in[-1,1]$, since $|\zeta| \leqslant 1$ as can be shown from Gershgorin's circle theorem [37]; see the Appendix. Using the boundedness of the $|\zeta|^{\prime}$ s and Eq. (9) we prove the proposition graphically; see Fig. 1 (left). The left-hand side (LHS) of Eq. (9) is quadratic in $\sigma$ and crosses the $x$-axis 

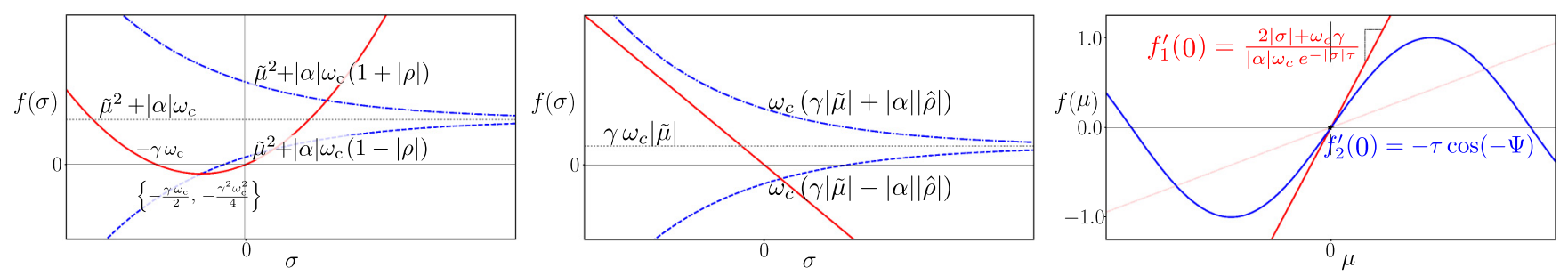

FIG. 1. Graphical solutions to Eqs. (7) shown for $\alpha=-|\alpha|<0$ (left), $\alpha>0, \tilde{\mu}<0$ (middle), and $\sigma \geqslant 0$, when $\tilde{\mu}=0$ (right). Red lines denote the LHS and blue the RHS of Eqs. (7), respectively.

at the origin $\sigma_{1}=0$ and at $\sigma_{2}=-\omega_{c} \gamma$. The right-hand side (RHS) of Eq. (9) crosses the $y$-axis, where $\tilde{\mu}=\mu(\sigma=0)$, at $\tilde{\mu}^{2}+|\alpha| \omega_{c}(1-\rho)$. Since $\rho \in[-1,1]$ the $y$-axis is always crossed at positive values if $\mu \neq 0$ and there is at least one intersection with $\sigma>0$ independently of the branches for $\pm \rho$. However, there could be an intersection at zero if $\mu=0$ and $\rho=1$. This occurs if $|\zeta| \cos (-\Psi)=1$, which is true only for $|\zeta|=1$ and $\Psi=2 \pi n(n \in \mathbb{Z})$, i.e., related to a global phase shift as previously discussed. This concludes the proof, and hence, for $\alpha<0$ there always exists at least one $\sigma>0$. Hence, the direction $\alpha<0 \rightarrow \sigma>0$ of the stability criterion in [35] holds in the presence of inertia.

Now we show that in regimes where the perturbation response dynamics are overdamped, i.e., $\mu=0$, the stability criterion holds also for second-order phase models. Hence, for $\mu=0$ and if $\alpha>0$ there can only be solutions with $\sigma<0$. Let us consider the contrary, for $\mu=0$ and if $\alpha>0$ there always exists at least one solution with $\sigma \geqslant 0$. In that case we would have $\mu=0, \sigma=|\sigma|$ and $\alpha=|\alpha|$. Using these expressions in Eq. (8) we find

$$
\frac{\left(|\sigma|^{2}+\omega_{c} \gamma|\sigma|+|\alpha| \omega_{c}\right)^{2}}{\left(|\alpha| \omega_{c}\right)^{2}}=|\zeta|^{2} e^{-2|\sigma| \tau} .
$$

The RHS is always in $[0,1]$ due to $|\zeta| \in[0,1]$ as shown before using Gershgorin's circle theorem and $e^{-2|\sigma| \tau} \in[0,1]$ for $\sigma \geqslant 0$. For $\sigma>0$ the LHS is always larger than 1 , which contradicts $|\zeta|^{2} e^{-2|\sigma| \tau} \leqslant 1$. The LHS can be equal to 1 only for $\sigma=0$, which leads to equality with the RHS for $\zeta= \pm 1$ only. For $\zeta=-1$ while $\lambda=0$ we know that $\alpha$ has to be zero [see Eq. (6)], which contradicts the assumptions. The case $\zeta=1$ relates to a global phase shift and is not considered as discussed before. As the contrary can never be fulfilled, the original proposition is always true.

Using the same graphical procedure as before in Fig. 1 (left) we now ask, for $\mu \neq 0$, whether if $\alpha>0$, there always exists at least one $\sigma \geqslant 0$. Setting $\alpha=|\alpha|$ in Eq. (7a) it can be shown that the proposition cannot always be fulfilled when studying the RHS for $\sigma=0$. If the asymptotic value of the RHS is $\tilde{\mu}^{2}-|\alpha| \omega_{c}<0$ and we consider the branch for $\rho>0$, then if $\tilde{\mu}^{2}-|\alpha| \omega_{c}(1-|\rho|)<0$ only solutions at $\sigma<$ 0 can exist. For $\rho<0$ there cannot be a solution for $\sigma \geqslant 0$ and a solution at $\sigma<0$ cannot be guaranteed. Hence, the proposition cannot always be fulfilled; bifurcations can occur when $\alpha>0$.

As we just showed, the synchronized states in Eq. (3) with constant phase difference 0 or $\pi$ are not guaranteed to be stable if $\alpha>0$ and $\mu \neq 0$. Hence, we proceed now to derive sufficient and necessary criteria that identify parameter regimes for which these states become unstable. Note that the parameters $\omega_{c}$ and $\gamma$ have a physical meaning and are positive. Studying the properties of Eqs. (7) at the critical point, $\sigma=0$, and taking into account their asymptotic properties for $\sigma \rightarrow \infty$, stability criteria that connect $\tilde{\mu}=\mu$ $(\sigma=0)$ and the parameters can be obtained. How the $\tilde{\mu}$ can be calculated explicitly is shown in the next section, which explains how the criteria derived here are applied. Using the information about the frequencies $\tilde{\mu}$ at the critical point we ask whether there are solutions to Eqs. (7) with positive real part $\sigma$. Rearranging Eq. (7b) and setting $\hat{\rho}=|\zeta| \sin (\mu \tau-\Psi)$ we find $2 \sigma \mu=-\omega_{c}\left(\mu \gamma+|\alpha| \hat{\rho} e^{-\sigma \tau}\right)$. Four cases $\{ \pm \tilde{\mu}, \pm \hat{\rho}\}$ need to be distinguished. The cases for $\tilde{\mu}=-|\tilde{\mu}|$ are shown in Fig. 1 (middle). Using the asymptotic property of the RHS of Eq. (7b) reveals that for the case $\alpha>0$ and $\hat{\rho}<0$ the RHS is bound to the first quadrant; see Fig. 1 (middle). At the same time the LHS of Eq. (7b) is bound to the fourth quadrant, and hence there cannot be an intersection for $\sigma \geqslant 0$. Using the same logic we now discuss the other case when $\alpha>0$ and $\hat{\rho}>0$. It can then be inferred that if $|\tilde{\mu}|>|\alpha||\hat{\rho}| / \gamma$ the RHS of Eq. (7b) intersects the $y$-axis in Fig. 1 (middle) above zero. Then no intersections can exist for $\sigma \geqslant 0$. Applying the same analysis for the other cases when $\tilde{\mu}=|\tilde{\mu}|$ one finds that for $\hat{\rho}>0$ there cannot be an intersection at $\sigma \geqslant 0$. For $\hat{\rho}<0$ no solutions at $\sigma \geqslant 0$ can exist if $|\tilde{\mu}|>|\alpha||\hat{\rho}| / \gamma$. The proof to conclude necessity has the same structure. Our criteria are in agreement with abstract mathematical results obtained for real $\zeta[38]$.

\section{APPLYING THESE CRITERIA TO STUDY LINEAR STABILITY}

The criteria derived in the last section can be meaningfully applied only if the $\tilde{\mu}$ are known. We calculate $\tilde{\mu}$ at the bifurcation, i.e., at the critical point $\sigma=0$. Hence, side bands at $\Omega \pm \tilde{\mu}$ arise in the power spectrum [19]. With these $\tilde{\mu}$ linear stability can be analyzed as a function of the network topology, interaction strength, damping coefficient, time delay, and inertial parameter. Moreover, we obtain a condition that predicts how the bifurcation can be prevented based only on the physical parameters of the system. Setting $\sigma=0$, while $\alpha=|\alpha|$, Eq. (8) after rearranging becomes

$$
\tilde{\mu}^{4}+\tilde{\mu}^{2}\left(\gamma^{2} \omega_{c}^{2}-2|\alpha| \omega_{c}\right)+\left(|\alpha| \omega_{c}\right)^{2}\left(1-|\zeta|^{2}\right)=0
$$


TABLE I. Stability criteria for $\alpha>0$ and $\mu \neq 0$.

\begin{tabular}{lc}
\hline \hline Sufficient and necessary criteria & Stability \\
\hline$\tilde{\mu}<0$ and $\hat{\rho}<0$ & Stable \\
$\tilde{\mu}<0$ and $\hat{\rho}>0$ and $|\tilde{\mu}|>|\alpha||\hat{\rho}| / \gamma$ & Stable \\
$\tilde{\mu}>0$ and $\hat{\rho}>0$ & \\
$\tilde{\mu}>0$ and $\hat{\rho}>0$ and $|\tilde{\mu}|>|\alpha||\hat{\rho}| / \gamma$ & Stable \\
$\zeta=-1$ and $\tilde{\mu}=0$ and $\gamma \geqslant|\alpha| \omega_{c}$ & \\
\hline \hline
\end{tabular}

Demanding $\tilde{\mu} \in \mathbb{R}$ while $\alpha>0$, a condition where synchronized states in Eq. (3) are stable is obtained:

$$
\frac{\omega_{c} \gamma^{2}}{2|\alpha|}>1-\sqrt{1-\left|\zeta_{0}\right|^{2}} .
$$

Here $\zeta_{0}$ denotes the eigenvalue with the largest magnitude. This result can be combined with the analysis of the criteria derived in the previous section using the solutions $\tilde{\mu}$ obtained from Eq. (11). For the case of $\zeta=-1(\Psi=\pi)$, e.g., the case for $N=2$ mutually coupled oscillators, the RHS and LHS of Eq. (7b) become zero when $\tilde{\mu}=0$. Such $\tilde{\mu}$ are actually solutions to Eq. (11) in this special case. This would imply that any type of perturbation response is a valid solution. Consulting Eq. (7a) for such $\tilde{\mu}=0$ when $\zeta=-1$ it becomes clear that additional information is necessary to infer whether or not the bifurcation has occurred. We need to plot Eq. (7b) for $\sigma \geqslant 0$ in the $\mu-f(\mu)$ plane and ask when additional solutions $\mu \neq 0$ can arise that lead to bifurcations; see Fig. 1 (right). From studying the slopes at $\mu=0$ and the smallest $|\sigma|=0$ we find that if $\gamma \geqslant|\alpha| \omega_{c}$ no additional solutions with $\sigma \geqslant 0$ can exist, and hence the state is linearly stable. In Tables I and II all criteria and conditions to be checked with which to study the linear stability of synchronized states are summarized. How these criteria and conditions are applied in practice to determine linear stability is explained in detail in the Appendix Sec. A 1.

Cyan denotes linearly unstable regimes where $\alpha<0$, and purple unstable regimes triggered by inert system behavior where $\alpha>0$. Gray specifies where the condition in Eq. (12) is fulfilled and the synchronized state is linearly stable. The stable regimes identified by the criteria in Table I are shown in white.

\section{PARAMETER SPACE PLOTS ANALYZING LINEAR STABILITY}

All parameter space plots share the same color code. When plots cover parameter space where multiple synchronized

TABLE II. Sufficient stability conditions.

\begin{tabular}{ll}
\hline \hline Sufficient conditions & Stability \\
\hline$\alpha<0$ & Unstable \\
$\alpha>0$ and $\mu=0$ & Stable \\
$\alpha>0$ and $1-\sqrt{1-\left|\zeta_{0}\right|^{2}}<\frac{\omega_{c} \gamma^{2}}{2|\alpha|}$ & Stable \\
\hline \hline
\end{tabular}

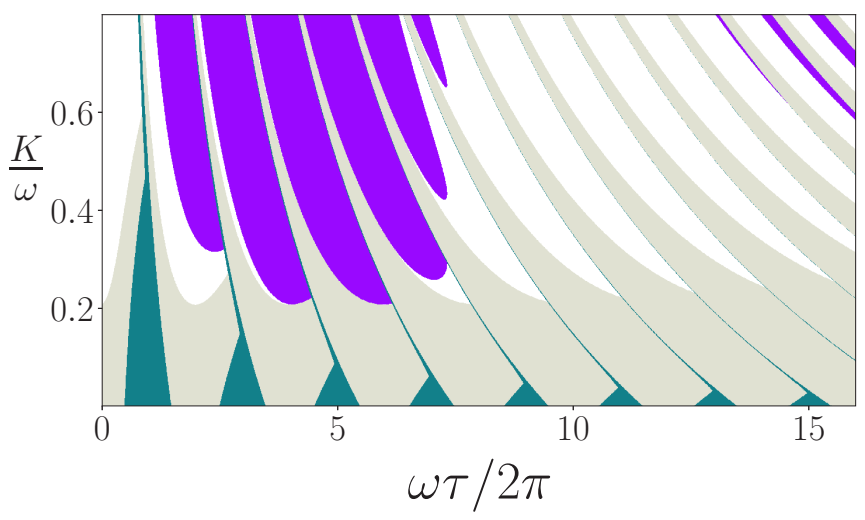

FIG. $2 . K$ vs $\tau$ parameter space for $3 \times 3$ identical oscillators mutually coupled to their nearest neighbors on a $2 \mathrm{D}$ square grid with periodic boundary conditions. Parameters are $\omega=2 \pi \mathrm{radHz}$, $v=1, \omega_{c}=0.028 \pi \mathrm{radHz}, \gamma=2, \zeta \in\{-0.5,0.25\}$. Cyan denotes linearly unstable regimes where $\alpha<0$ and purple unstable regimes triggered by inert system behavior where $\alpha>0$. Gray specifies where the condition in Eq. (12) is fulfilled and the synchronized state is linearly stable. The linearly stable regimes identified by the criteria in Table I are shown in white.

states are stable, the stability of the one with the largest frequency $\Omega=\left\{\Omega_{i}\right\}_{i \in \mathbb{N}}^{\max }$ is plotted. Python scripts that implement these criteria are available online [39]. These can also solve Eq. (6) numerically for validation purposes; see examples provided in the Appendix Sec. A 3. For all parameter space plots presented in this work we consider a sinusoidal coupling function $h(\cdot)$.

Cyan denotes regimes where $\alpha<0$ and the in- or antiphase synchronized state [see Eq. (3)] is unstable. States with different constant phase relations exist in these regimes and can be stable if $\alpha>0$; see Fig. 11 in the Appendix Sec. A 3 c. Purple regimes denote where in- or antiphase synchronized states are unstable due to inert system behavior when $\alpha>0$. They are qualitatively different from parameter regimes where synchronized states that satisfy Eq. (3) become unstable when $\alpha<0$. At their onset they are characterized by time-dependent frequencies and highly correlated periodic dynamics; see Figs. 12 and 13 in the Appendix Sec. A 3 c. Hence, synchronization in a wider sense is not necessarily lost after the Hopf bifurcation. There are indications that these systems undergo a route to chaos via subsequent perioddoubling bifurcations as, e.g., the time delay is increased [40].

Stable synchronized states are shown in gray and white. Gray specifies where the condition in Eq. (12) is fulfilled for the $\zeta_{0}$ with the largest magnitude. In general, the synchronized states of Eq. (3) tend to become unstable as the oscillators become increasingly inert $\left(\omega_{c} \ll \omega\right)$, and the purple regimes cover larger areas in Fig. 2. Since $\omega_{c}$ plays an important role in suppressing higher order frequency contributions in real systems, it cannot be increased freely. Above some critical $\omega_{c}$ the presence of, e.g., intermodulation products which are not described in Eq. (1) can also lead to timedependent frequencies. Our results can guide towards optimal parameter choices for applications, e.g., in coupled electronic oscillators. 


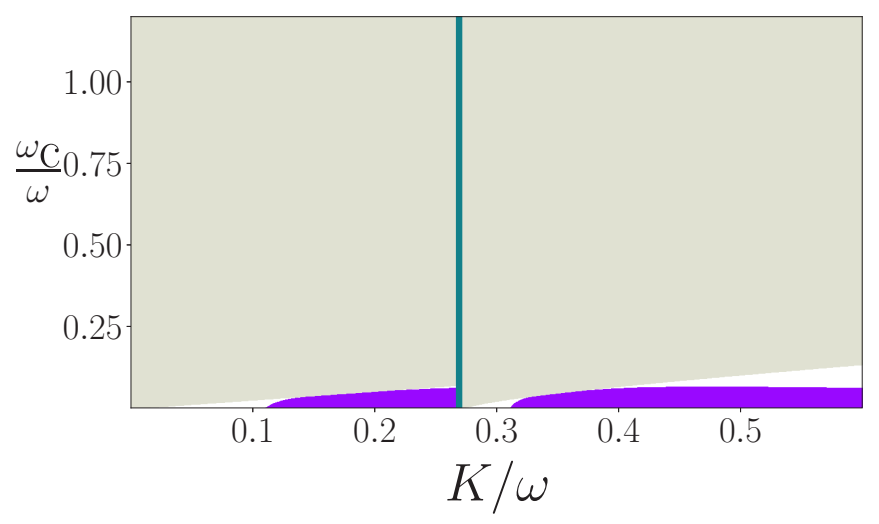

FIG. 3. $\omega_{c}$ vs $K$ parameter space. Parameters, network topology, and color code as in Fig. 2, except $\gamma=1$ and $\tau=0.65 \mathrm{~s}$.

\section{THE PHYSICS OF SYNCHRONIZATION FOR LARGE DELAYS}

The stability in the presence of large time delays has been studied using asymptotic descriptions [41-45]. Simple and explicit expressions for the linear stability can then be obtained in the limit of large or infinite time delay. In this section we study networks in which the time delays are finite but large compared to the intrinsic period of the oscillators. Our analysis does not produce quantitative results like perturbation decay rates, but instead provides the information whether or not a synchronized state is linearly stable. Networks of mutually coupled electronic oscillators, so-called phase-locked loops [46], are candidates for enabling new technologies, such as satellite-independent terrestrial navigation, and to provide orchestration to complex spatially distributed systems. Their function relies on a robust clock signal distribution. Given operational frequencies up to the $\mathrm{THz}$ regime, spatial extensions of a few hundreds of meters imply time delays that are three to six orders of magnitude larger than the oscillation period. In these cases synchronization can be stable only for adequately divided cross-coupling frequencies [47]. This also requires one to sufficiently decrease $\omega_{c}$, i.e., making the oscillators more inert. Otherwise side bands will appear in the frequency spectrum that may lead to, e.g., cross-channel interference [48]. In consequence, the loop gains $\alpha$ have to be tuned sufficiently small to prevent violation of the condition in Eq. (12) as $\omega_{c}$ is decreased. Our results also show that $\omega_{c}$ can be optimized beyond this condition; see white spaces in Fig. 3. Another challenge is the large number of synchronized states that can exist simultaneously. As a result, it becomes difficult to determine stability numerically or in simulations. Using the criteria we derived, the stability at arbitrary time delays can now be obtained. We find that synchronization is feasible even when time delays span thousands of the oscillators' periods; see Fig. 4. It shows that there are stable synchronized states (gray) for sufficiently small coupling strength $K$. The condition in Eq. (12) involves a periodic dependence on the time delay via $\alpha$. This suggests that fine tuning the delay can enable stable synchronization at very large time delay. In real systems this may be limited by signal degradation during sending and dynamic noise. Note also that for $N$ all to all (globally) coupled oscillators $\zeta_{0}=(N-1)^{-1}$. Hence,

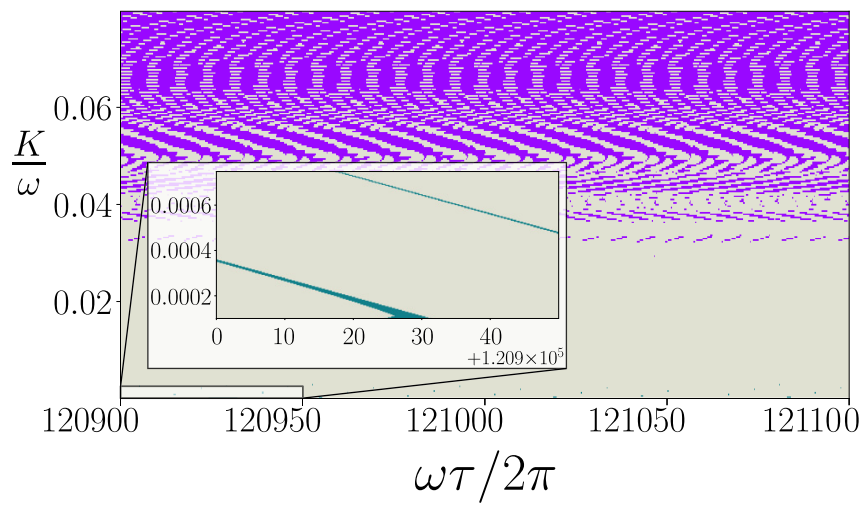

FIG. 4. $K$ vs $\tau$ parameter space for $3 \times 3$ identical oscillators with nearest-neighbor coupling on a $2 \mathrm{D}$ square grid with open boundary conditions. Parameters are $\omega=2 \pi \mathrm{radHz}, \gamma=1, v=64, \omega_{c}=$ $0.0007 \pi \mathrm{radHz}$, and $\zeta \in\{-1,-0.5,0.5\}$. Cyan structures in inset are not visible in main due to resolution. The color code is identical to Fig. 2.

the stable regime guaranteed by condition Eq. (12) increases with $N$.

\section{DAMPING COEFFICIENT RESCALES DELAY AND FREQUENCY}

$\gamma$ relates to, e.g., gains in electronic oscillators, a friction in mechanical or damping coefficient in power grid systems. Substituting $\Omega^{*}=\gamma \Omega$ and $\tau^{*}=\tau / \gamma$ in Eq. (3) reveals that $\gamma$ acts as a rescaling of the time delay and frequency of synchronized states. The relation between time delay and period of the oscillations changes; observe the repetitive cyancolored structures where $\alpha<0$ in Fig. 5. Decreasing $\gamma$ below one increases the frequency $\Omega$ of a synchronized state for constant $\omega_{c}$ that changes the ratio $\omega_{c} / \Omega$ and can trigger inertia-induced bifurcations. The rescaling of the time delay by the damping coefficient can also be seen in Fig. 6. This may have implications for systems that rely on specific synchronized states between spatially distributed oscillators, e.g., the periodic swimming strokes of hydrodynamically coupled cilia of the green alga Chlamydomonas [49]. Environmental

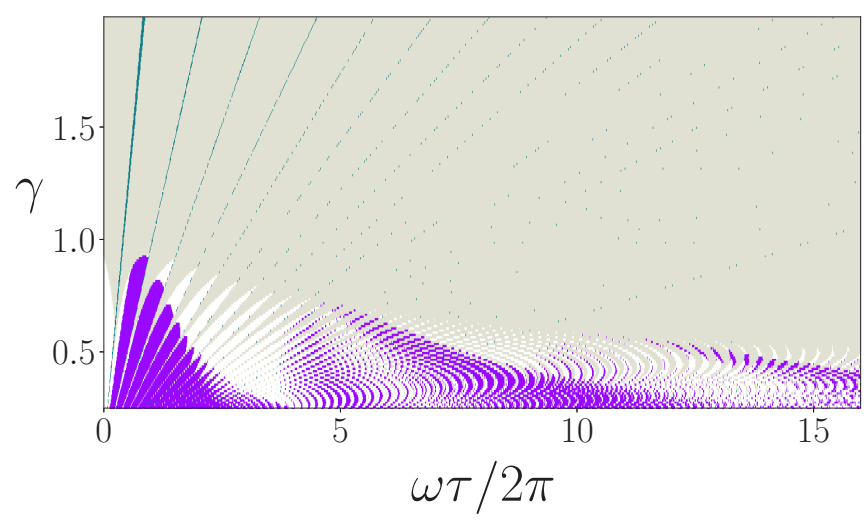

FIG. 5. $\gamma$ vs $K$ parameter space. Parameters, network topology, and color code as in Fig. 2, except $\omega_{c}=0.2 \pi$ and $\tau=2.95 \mathrm{~s}$. 


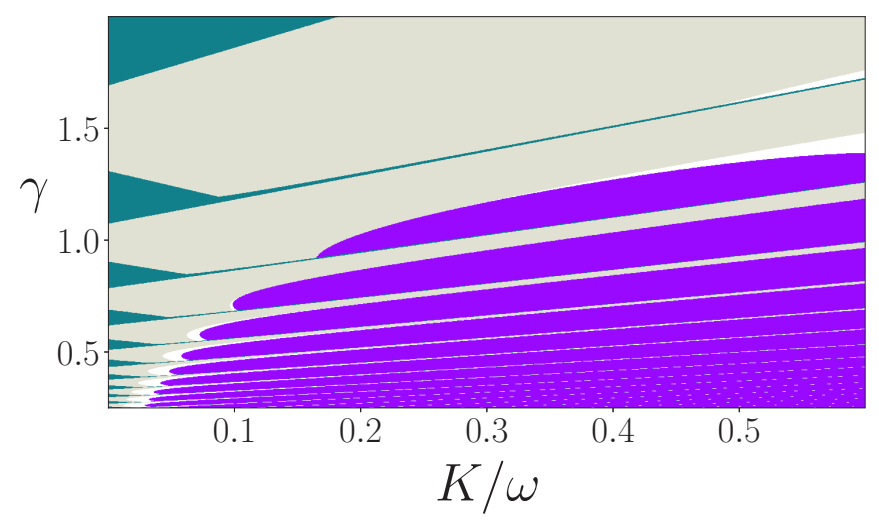

FIG. 6. $\gamma$ vs $\tau$ parameter space. Parameters, network topology, and color code as in Fig. 2, except $\omega_{c}=0.4 \pi \mathrm{radHz}$ and $K=1.3 \pi \mathrm{radHz}$.

conditions that alter the damping coefficient $\gamma$ could hence lead to changes in the phase relations between cilia beats.

\section{DISCUSSION AND CONCLUSIONS}

We derived general stability criteria for in- and antiphase synchronized states in systems of delay-coupled oscillators with inertia. Using them, we identify parameter regimes where systems with inert oscillator response can excite additional frequencies or lead to chaotic dynamics. In a synchronized state with $\{\Omega, \beta\}$ constant in time, $\alpha$ denotes the change of the oscillators interaction terms with respect to a small perturbation. For $\alpha<0$ the interaction between the oscillators becomes repelling. In this case another stable synchronized state exists for which $\alpha>0$ and the coupling is attractive. As long as perturbation responses are overdamped no bifurcations occur. That changes for an underdamped response. Then our criteria and condition (12) reveal how the interplay between the oscillators' parameters and those of the network lead to bifurcations. A specific example is how the cutoff frequency $\omega_{c}$ of a filter limits the loop gain $\alpha$ in networks of electronic oscillators; see condition (12). In the presence of dynamic noise, this analysis can be carried out within the Fokker-Planck formalism [50,51] and is subject to ongoing work.

Our analysis can be applied to various fields as the response of natural systems usually is inert $[2,7,11]$. It is especially helpful when the numerical solution of the characteristic Eq. (6) or simulations become infeasible. For applications, our results enable fast identification of the parameter regimes where synchronized states with constant phase differences are stable. This will improve the architecture design process of, e.g., networks of electronic oscillators [11,32]. Furthermore it can enable real-time control algorithms for on-the-fly optimization of such complex systems, e.g., when topology or time delays change over time. We show that stable mutual synchronization is feasible at large time delay. This makes it a candidate for the next generation self-organized clocking signal distribution layers [52]. It is relevant for, e.g., precise localization using microsatellites or terrestrial beacons, sensoring and time distribution, and high-precision physical measurements in spatially distributed systems such as very long baseline interferometry and gravitational wave detection [53-56].

\section{ACKNOWLEDGMENTS}

We would like to pay our regards to C. Zheng, P. Pnigouras, H. Kantz, D. Schmidt, P. Giannakeas, D. Jörg, A. Pollakis, G. Fettweis, and F. Jülicher for inspiring discussions. The work on this topic was initiated within the Cluster of Excellence Center for Advancing Electronics Dresden. This work was supported by the Federal Ministry of Education and Research (BMBF) under the Reference Number 03VP06431.

D.P. and L.W. contributed equally to this work.

\section{APPENDIX:}

Here we provide technical details on the calculations and theorems used in the main text. We present a numerical verification of the conditions and criteria presented, using parameter plots obtained from solving the characteristic equation numerically. In addition we show exemplary time series plots of the frequencies and phase relations for the different types of states in the parameter space plots. The criteria are summarized in a table for convenient application. The parameters of all plots in the the paper and the Appendix are listed in Tables III and IV.

\section{How to apply the critera and conditions}

In a first step the properties of the synchronized states whose linear stability is to be studied have to be computed using

$$
\gamma \Omega=\omega+K h\left(-\frac{\Omega \tau+\beta}{v}\right) .
$$

This yields the global frequencies $\Omega$ and phase differences $\beta$ of the existing synchronized states. For these solutions, the parameters

$$
\alpha(\Omega, \beta)=\frac{K}{v} h^{\prime}\left(\frac{-\Omega \tau+\beta}{v}\right)
$$

can be obtained. Linear stability needs to be studied for each of these solutions individually. In a next step the eigenvalues $\zeta$ of the normalized adjacency matrix need to be calculated. Then solving Eq. (11) yields $\tilde{\mu}$ from

$$
\tilde{\mu}= \pm \sqrt{-\frac{A}{2} \pm \frac{A}{2} \sqrt{1-\frac{4 B}{A^{2}}}}
$$

where $\alpha>0, A\left(\tau, K, \omega_{c}, \gamma, v\right)=\gamma^{2} \omega_{c}^{2}-2|\alpha| \omega_{c}$ and $B(\tau, K$, $\left.\omega_{c}, \zeta, \gamma, v\right)=\left(|\alpha| \omega_{c}\right)^{2}\left(1-|\zeta|^{2}\right)$. The stability analysis using the criteria in Table I requires one to compute the $\tilde{\mu}$ explicitly from Eq. (A3) for a given set of parameters. Note that complex valued $\tilde{\mu}$ contradict the definition, and the stability for such $\tilde{\mu}$ may not be found using the criteria. For such complex $\tilde{\mu}$ a theorem [57] allows one to infer linear stability if $\zeta \in \mathbb{R}$. It shows that no solutions $\lambda$ have crossed the imaginary axis to the RHS if no $\tilde{\mu} \in \mathbb{R}$ exist. Then demanding that $\tilde{\mu} \in \mathbb{R}$ we obtain from Eq. (A3) a sufficient condition that, if fulfilled 
TABLE III. Parameter values of the in plots in main text.

\begin{tabular}{|c|c|c|c|c|c|c|c|}
\hline Fig. & $\begin{array}{c}\omega \\
(\operatorname{radHz})\end{array}$ & $\begin{array}{c}\omega_{c} \\
(\operatorname{radHz})\end{array}$ & $\begin{array}{c}\tau \\
(\mathrm{sec})\end{array}$ & $\begin{array}{c}\gamma \\
(\operatorname{radHz})\end{array}$ & $\begin{array}{c}K \\
(\operatorname{radHz})\end{array}$ & $\zeta$ & $v$ \\
\hline 2 & $2 \pi$ & $0.028 \pi$ & - & 2 & - & $\{-0.5,0.25\}$ & 1 \\
\hline 3 & $2 \pi$ & $0.0007 \pi$ & - & 1 & - & $\{-1,-0.5,0.25\}$ & 64 \\
\hline 4 & $2 \pi$ & - & 0.65 & 1 & - & $\{-0.5,0.25\}$ & 1 \\
\hline 5 & $2 \pi$ & $0.4 \pi$ & - & - & $1.3 \pi$ & $\{-0.5,0.25\}$ & 1 \\
\hline 6 & $2 \pi$ & $0.2 \pi$ & 2.95 & - & - & $\{-0.5,0.25\}$ & 1 \\
\hline
\end{tabular}

while $\alpha>0$ and $\zeta \in \mathbb{R}$, guarantees linear stability:

$$
\frac{\omega_{c} \gamma^{2}}{2|\alpha|}>1-\sqrt{1-\left|\zeta_{0}\right|^{2}} .
$$

This condition (A4) can then be used to understand where synchronized states must be stable. If this condition is not fulfilled and $\tilde{\mu} \in \mathbb{R}$ exist, the criteria in Table I identify for which parameters states with time-dependent frequencies have emerged from the ones with constant frequencies. These criteria also hold for $\zeta \in \mathbb{C}$ and follow from the analysis presented in Sec. III that takes into account the asymptotic behavior for $\sigma \rightarrow \infty$ and at the critical point $\sigma=0$, the latter of which is associated with $\tilde{\mu}$. Hence, for each eigenvalue $\zeta$ of the adjacency matrix all associated $\tilde{\mu}$ calculated explicitly from Eq. (A3) for the parameter set $\left\{\tau, K, \omega_{c}, \gamma, v\right\}$ need to be checked. If for all $\tilde{\mu}$ the necessary and sufficient criteria in Table I are fulfilled, no solutions $\lambda$ to the characteristic Eq. (6) with $\sigma>0$ exist. Hence, the synchronized state associated with the parameter set $\{\tau, K, \gamma, v\}$ is stable. All criteria and conditions are summarized in Tables I and II and need to be checked in order to study the linear stability of in- and antiphase synchronized states with constant frequency. Note that when using the Python scripts for the parameter space plots the eigenvalues are specified as pairs $\{|\zeta|, \Psi\}$ since $\zeta=|\zeta| \exp (i \Psi)$

\section{Gershgorin theorem}

The Gershgorin theorem defines disks centered around each diagonal entry of a matrix $\mathbb{D}$ with radius given by the row sum over all nondiagonal entries [37]. As there is no self-coupling all $d_{k k}=0 \forall k$, and hence all Gershgorin discs are centered at the origin. Hence $|\zeta|$ is bounded by one:

$$
|\zeta| \leqslant \sum_{l \neq k}\left|d_{k l}\right|=1 .
$$

Taking into account the definition $d_{k l}=c_{k l} / n_{k}$, the boundedness of the $\zeta$ given by Gershgorin's theorem is tied to the identical coupling capacity of all oscillators

$$
\frac{1}{n_{k}} \sum_{l \neq k}\left|c_{k l}\right|=1 .
$$

This can also be achieved when the oscillators weight different inputs with different weights,

$$
\frac{1}{n_{k l}} \sum_{l \neq k}\left|c_{k l}\right|=1 \text {. }
$$

when the sum over all nondiagonal entries equals one.

\section{Additional plots and numerical verification}

The parameter space plots, Figs. 8 and 9, share the same color code as introduced in Sec. V. The numerical results

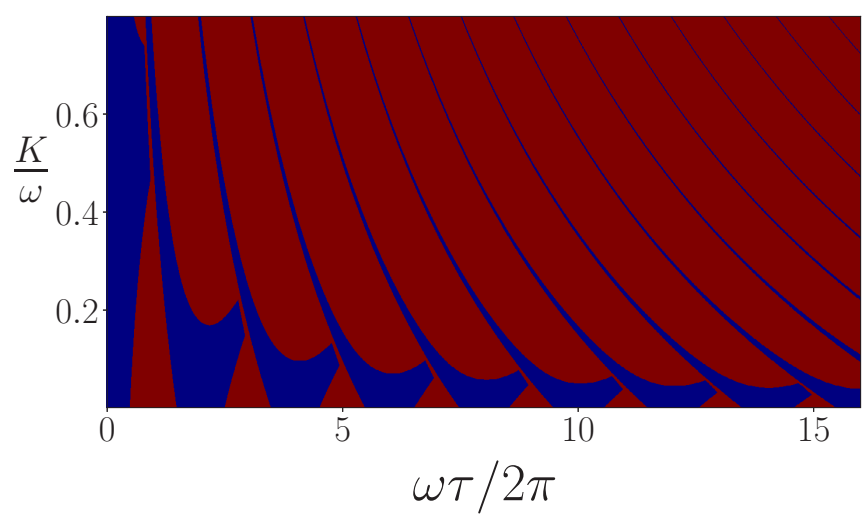

FIG. 7. $K$ vs $\tau$ parameter space for $N=2$ mutually delaycoupled oscillators, obtained numerically. All other parameters are listed in Table IV. Red denotes regimes where in-phase synchronizes states are unstable, blue where they are stable.

TABLE IV. Parameters of plots in the Appendix.

\begin{tabular}{lcccccr}
\hline \hline Fig. & $\begin{array}{c}\omega \\
(\mathrm{radHz})\end{array}$ & $\begin{array}{c}\omega_{c} \\
(\mathrm{radHz})\end{array}$ & $\begin{array}{c}\tau \\
(\mathrm{sec})\end{array}$ & $\begin{array}{c}\gamma \\
(\mathrm{radHz})\end{array}$ & $\begin{array}{c}K \\
(\mathrm{radHz})\end{array}$ & $\zeta$ \\
\hline 7,8 & $2 \pi$ & $0.028 \pi$ & - & 2 & - & -1 \\
9 & $2 \pi$ & $0.028 \pi$ & - & 1 & 0.2 & -1 \\
10 & $2 \pi$ & $0.028 \pi$ & 0.1 & 1 & 0.2 & -1 \\
11 & $2 \pi$ & $0.028 \pi$ & 0.7 & 1 & 0.2 & -1 \\
12 & $2 \pi$ & $0.028 \pi$ & 5 & 1 & - & 1 \\
13,14 & $2 \pi$ & $0.2 \pi$ & 2.95 & - & $-0.5,0.25\}$ \\
\hline \hline
\end{tabular}




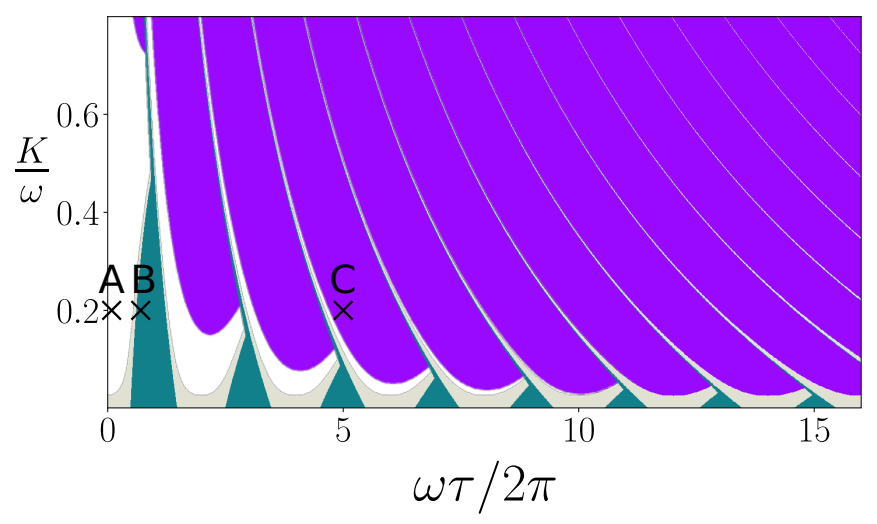

FIG. 8. $K$ vs $\tau$ parameter space for $N=2$ mutually delaycoupled oscillators, obtained analytically via the criteria and condition. All other parameters listed in Table IV. The analytic results are verified with Fig. 7. Exemplary time series at points $A, B$, and $C$ are shown in Figs. 10-12. The color code is identical to Fig. 2.

in Fig. 7 have a different color code as introduced in the following subsection.

\section{a. Numerical verification of the criteria}

We verify the results obtained using the criteria and the conditions with numerical solutions to the characteristic equation. The numerical results are obtained using scripts which are available online [39]. The parameters of the system under investigation can be set in the dictionaries of these scripts. The numerically obtained parameter space plot in Fig. 7 has the following color code. In this case red denotes regimes

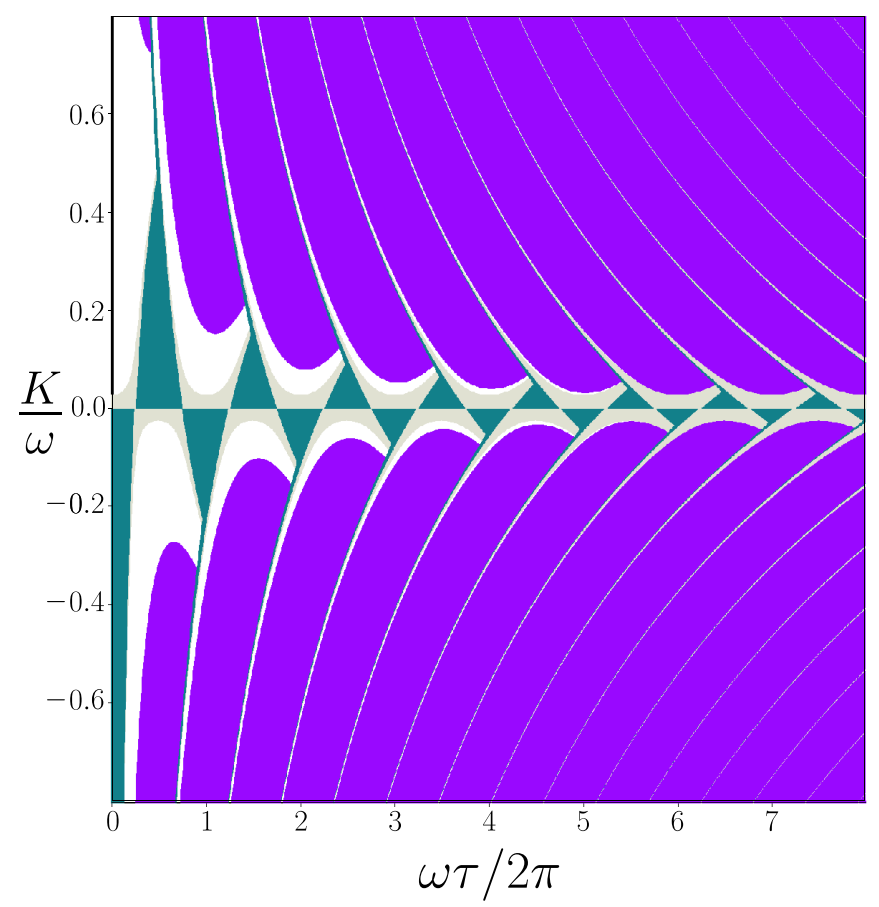

FIG. 9. $K$ vs $\tau$ parameter space for $N=2$ mutually delaycoupled oscillators, obtained analytically via the criteria and condition. In- $(K \geqslant 0)$ and antiphase $(K<0)$ synchronized states are shown. All other parameters listed in Table IV. The color code is identical to Fig. 2.

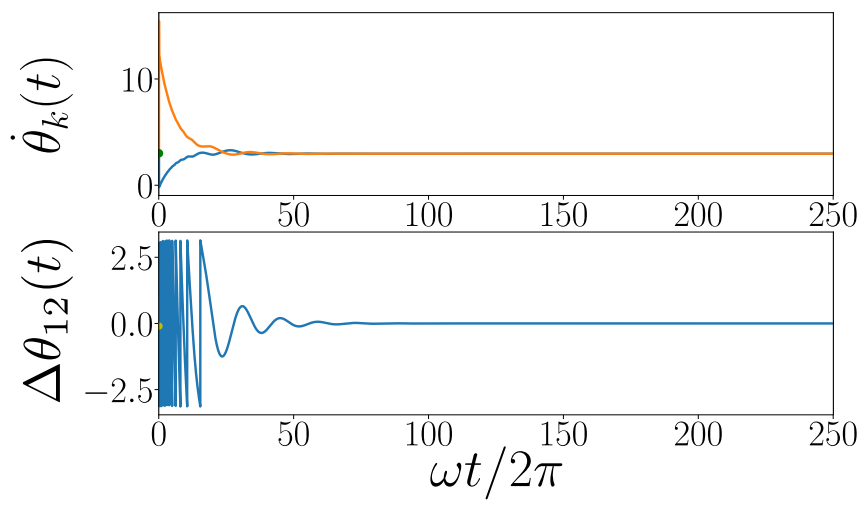

FIG. 10. Time series of the instantaneous frequencies $\dot{\theta}_{k}(t)$ and the phase difference $\Delta \theta_{12}(t)$ for case A in Fig. 8. This is the in-phase synchronized state with Kuramoto order parameter equal to one.

where the real part $\sigma$ of the characteristic equation is positive, and hence the synchronized states are unstable. Blue denotes regimes where $\sigma$ is negative, and in- and/or antiphase synchronized states are stable. We choose this different color code for the numerical results to avoid confusion and clearly distinguish them from those obtained with the stability conditions and criteria.

It becomes apparent from the comparison of Figs. 7 and 8 that the results obtained analytically using the criteria are in agreement with the numerical results. The value of the perturbation response rate obtained from the numerical solution shows no qualitative difference for the white and gray regimes. Hence, it is not represented by the color code in Fig. 7. Also, the exemplary time series obtained from simulating Eqs. (1) using an Euler scheme confirm the predictions using the conditions.

\section{b. Parametric plots of different topologies}

Here we show parametric plots for different network topologies of mutually delay-coupled oscillators with inertia. All other parameters have the same values, i.e., identical oscillator parameters and time delays.

In Fig. 8 the $K-\tau$ parameter space for a network of two mutually coupled oscillators is shown. The nontrivial eigenvalue

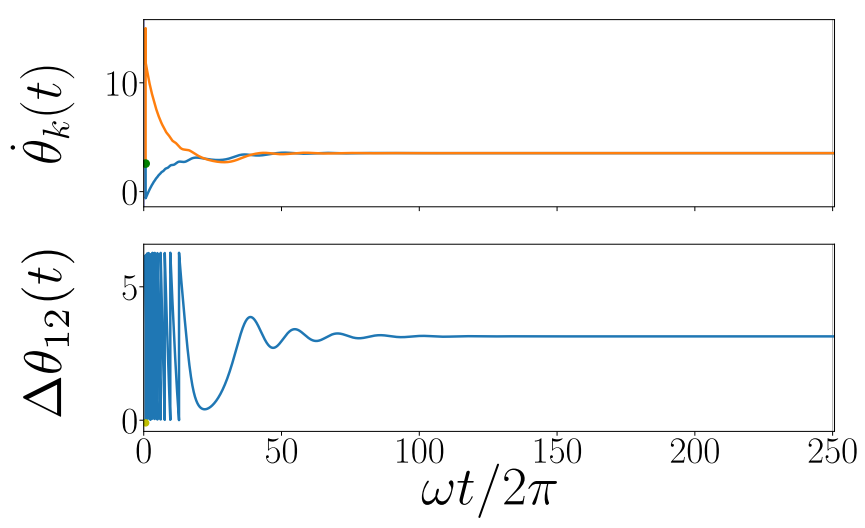

FIG. 11. Time series of the instantaneous frequencies $\dot{\theta}_{k}(t)$ and the phase difference $\Delta \theta_{12}(t)$ for case B in Fig. 8 . This is the antiphase synchronized state with Kuramoto order parameter equal to zero. 

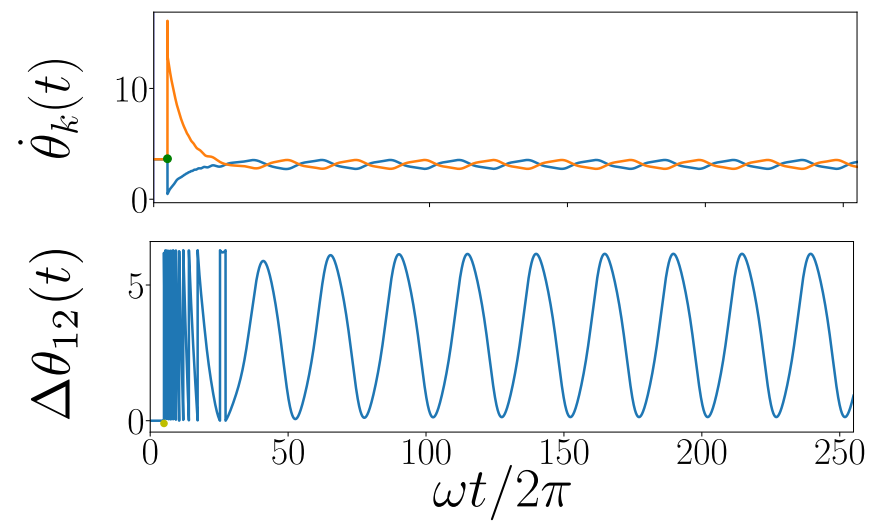

FIG. 12. Time series of the instantaneous frequencies $\dot{\theta}_{k}(t)$ and the phase difference $\Delta \theta_{12}(t)$ for case $\mathrm{C}$ in Fig. 8.

$\zeta$ of the adjacency matrix is $\zeta=-1$. In Fig. 2 the parameter space of a network of nine mutually delay-coupled oscillators in a 2D lattice with nearest-neighbor interactions and periodic boundary conditions is shown. The nontrivial eigenvalues $\zeta$ of the adjacency matrix in this case are $\zeta=\{-0.5,0.25\}$. It can be observed that linear stability is different for the two cases. The condition in Eq. (A4) is fulfilled for different regimes, shown in gray in the figures. When $\zeta=-1$ then gray covers the smallest regime, as can be understood from Eq. (A4). For networks in which the oscillators are arranged in $1 \mathrm{D}$ with open boundary conditions, e.g., a chain topology, the value $\zeta=-1$ is always in the set of $\zeta$ [19]. The criteria which are presented in Table I are also affected by the topology, and hence the stability regimes in white and purple are also different.

\section{c. Parametric plot of in- and antiphase synchronized states}

In Fig. 9 we show the parametric $K-\tau$ plot for in- and antiphase synchronized states in a system of two mutually delay-coupled oscillators with inertia. The regime for $K>0$ relates to the in-phase synchronized states and for $K<0$ to the antiphase synchronized states; see also [35]. Observe how the cyan regimes alternate between in- and antiphase synchronized state as the time delay increases.

\section{d. Time series data}

The complex order parameter is a quantity with which phase synchronization can be quantified [13]

$$
R(t) e^{i \psi(t)}=\frac{1}{N} \sum_{k=1}^{N} e^{i \theta_{k}(t)}
$$

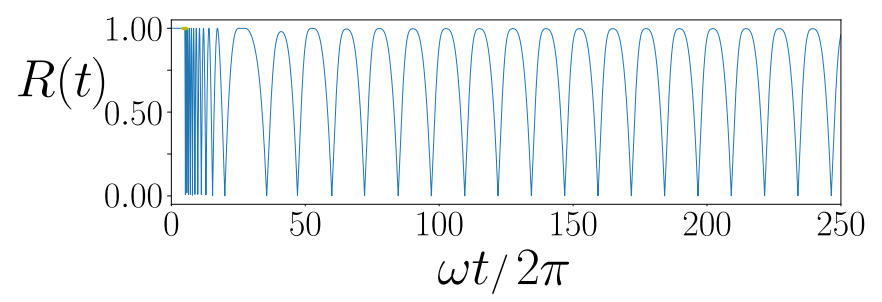

FIG. 13. Time series of the order parameter $R(t)$ for case $\mathrm{C}$ in Fig. 8. The instantaneous frequencies $\dot{\theta}_{k}(t)$ and the phase difference $\Delta \theta_{12}(t)$ are shown in Fig. 12.

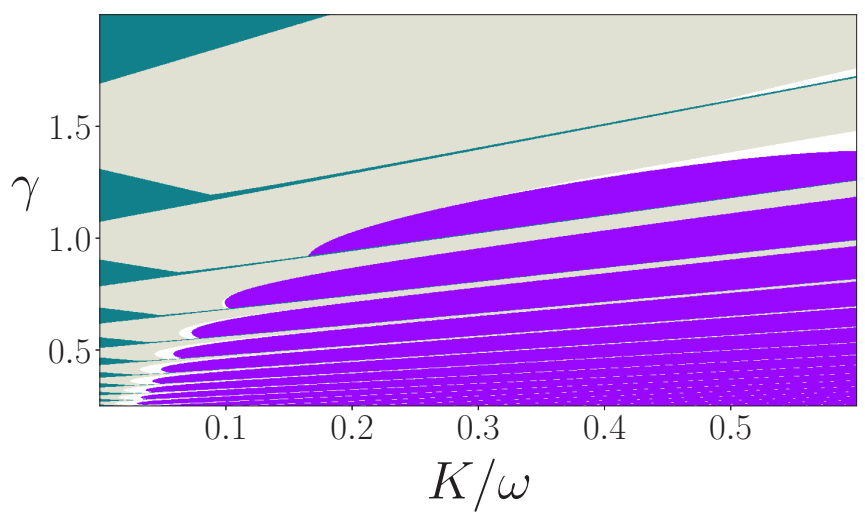

FIG. 14. $K$ vs $\gamma$ parameter space for $3 \times 3$ mutually delaycoupled oscillators on a $2 \mathrm{D}$ square grid with periodic boundary conditions, obtained analytically via the criteria and condition for $\Omega$ obtained with Eq. (A1). All other parameters listed in Table IV. The color code is identical to Fig. 2.

$R(t)$ measures the phase coherence and $\psi(t)$ denotes the average phase. In Figs. 10-12 we show the time series of the phase difference between and the frequencies of two mutually delay-coupled oscillators with inertia for different sets of parameters. In Fig. 13 the time series of the order parameter for $\tau=5$, i.e., case $C$ in Fig. 8, is presented. The in-phase (see Fig. 10) and the antiphase synchronized states (see Fig. 11) are stable with respect to a small initial phase perturbation. Rich system dynamics can be observed where synchronized states become linearly unstable due to the presence of the inertia. Close to the bifurcation, a stable limit cycle appears, and hence the frequency of the oscillator becomes periodic; see Fig. 12. Hence, the time series plots show the expected solutions for the different regimes in Fig. 8. Note that more examples of time series of solutions that bifurcate from inand antiphase synchronized states in the purple regimes can be found in the supplemental materials of Punetha et al. [40].

\section{A note on the damping coefficient}

Here we want to make the reader aware of a small difference between models of mutually delay-coupled oscillators

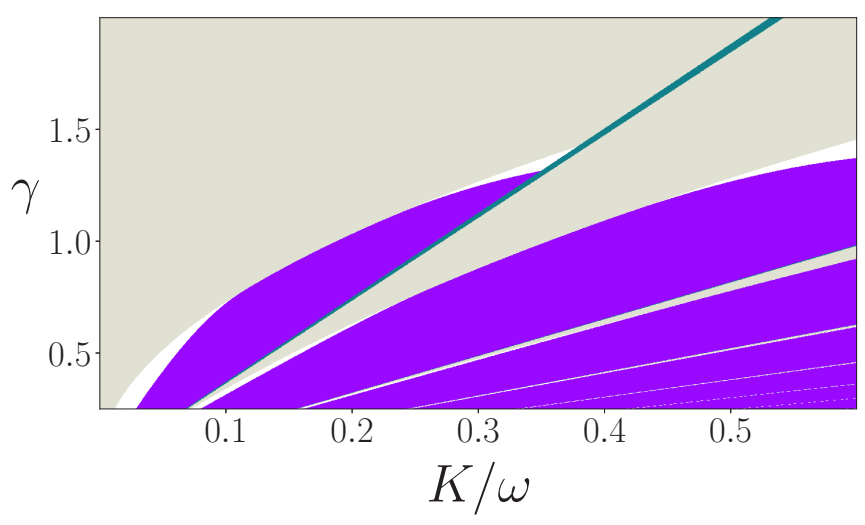

FIG. 15 . $K$ vs $\gamma$ parameter space for $3 \times 3$ mutually delaycoupled oscillators on a $2 \mathrm{D}$ square grid with periodic boundary conditions, obtained analytically via the criteria and condition for $\Omega$ obtained with Eq. (A10). All other parameters listed in Table IV. The color code is identical to Fig. 2. 
that have been presented previously $[8,58]$. Besides the dynamical Eqs. (1), there are also other formulations, e.g., for a system of coupled rotors $[58,59]$

$$
m \ddot{\theta}_{k}(t)+\gamma \dot{\theta}_{k}(t)=\gamma \omega+\frac{K}{n_{k}} \sum_{l=1}^{N} c_{k l} h\left(\frac{\Delta \theta_{k l}(t, \tau)}{v}\right) .
$$

In this case the damping coefficient affects the frequency of the free running oscillator, contrary to the case described by
Eqs. (1). For the properties of synchronized states one finds

$$
\gamma \Omega=\gamma \omega+K h\left(-\frac{\Omega \tau+\beta}{v}\right) .
$$

The frequencies of synchronized states are hence different from those obtained using Eq. (3). Since the steady-state parameter $\alpha$ depends on the frequency $\Omega$ linear stability is affected as well. In Figs. 14 and 15 the difference in linear stability between the two different models is presented.
[1] J. Buck, Q. Rev. Biol. 63, 265 (1988).

[2] B. R. Trees, V. Saranathan, and D. Stroud, Phys. Rev. E 71, 016215 (2005).

[3] V. Flunkert, S. Yanchuk, T. Dahms, and E. Schöll, Phys. Rev. Lett. 105, 254101 (2010).

[4] A. C. Oates, L. G. Morelli, and S. Ares, Development 139, 625 (2012).

[5] M. Rohden, A. Sorge, M. Timme, and D. Witthaut, Phys. Rev. Lett. 109, 064101 (2012).

[6] A. Motter, S. Myers, M. Anghel, and T. Nishikawa, Nat. Phys. 9, 191 (2013).

[7] T. Dewenter and A. K. Hartmann, New J. Phys. 17, 015005 (2015).

[8] F. A. Rodrigues, T. K. D. Peron, P. Ji, and J. Kurths, Phys. Rep. 610, 1 (2016).

[9] N. V. Kuznetsov, G. A. Leonov, M. V. Yuldashev, and R. V. Yuldashev, arXiv:1705.05013.

[10] E. Koskin, D. Galayko, and E. Blokhina, IEEE Access 6, 18723 (2018).

[11] W. C. Lindsey, F. Ghazvinian, W. C. Hagmann, and K. Dessouky, Proc. IEEE 73, 1445 (1985).

[12] C. C. Santini and A. Tyrrell, IEEE Trans. NanoBiosci. 8, 237 (2009).

[13] Y. Kuramoto, Prog. Theor. Phys. Suppl. 79, 223 (1984).

[14] D. J. Jörg, A. Pollakis, L. Wetzel, M. Dropp, W. Rave, F. Jülicher, and G. Fettweis, in Proceedings of the 2015 IEEE International Conference on Communications (ICC) (IEEE, London, UK, 2015), pp. 1716-1721.

[15] H. G. Schuster and P. Wagner, Prog. Theor. Phys. 81, 939 (1989).

[16] S. Gupta, A. Campa, and S. Ruffo, J. Stat. Mech. (2014) R08001.

[17] S. Olmi, A. Navas, S. Boccaletti, and A. Torcini, Phys. Rev. E 90, 042905 (2014).

[18] S. M. L. O. Diekmann, S. A. van Gils, and H.-O. Walther, Applied Mathematical Sciences (Springer-Verlag, New York, 1993).

[19] A. Pollakis, L. Wetzel, D. J. Jörg, W. Rave, G. Fettweis, and F. Jülicher, New J. Phys. 16, 113009 (2014).

[20] J. Sieber, K. Engelborghs, T. Luzyanina, G. Samaey, and D. Roose, arXiv:1406.7144.

[21] L. M. Pecora and T. L. Carroll, Phys. Rev. Lett. 80, 2109 (1998).

[22] K. S. Fink, G. Johnson, T. Carroll, D. Mar, and L. Pecora, Phys. Rev. E 61, 5080 (2000).

[23] A. Arenas, A. Díaz-Guilera, J. Kurths, Y. Moreno, and C. Zhou, Phys. Rep. 469, 93 (2008).
[24] T. Dahms, J. Lehnert, and E. Schöll, Phys. Rev. E 86, 016202 (2012).

[25] J. Lehnert, T. Dahms, P. Hövel, and E. Schöll, Europhys. Lett. 96, 60013 (2011).

[26] J. D. Hart, J. P. Pade, T. Pereira, T. E. Murphy, and R. Roy, Phys. Rev. E 92, 022804 (2015).

[27] S. R. Huddy, Chaos 30, 013118 (2020).

[28] R. Börner, P. Schultz, B. Ünzelmann, D. Wang, F. Hellmann, and J. Kurths, Phys. Rev. Res. 2, 023409 (2020).

[29] G. Filatrella, A. Nielsen, and N. Pedersen, Eur. Phys. J. B 61, 485 (2008).

[30] B. Schäfer, M. Matthiae, M. Timme, and D. Witthaut, New J. Phys. 17, 015002 (2015).

[31] L. R. Gorjão, M. Anvari, H. Kantz, C. Beck, D. Witthaut, M. Timme, and B. Schäfer, IEEE Access 8, 43082 (2020).

[32] L. Wetzel, D. Prousalis, R. Riaz, C. Hoyer, N. Joram, J. Fritzsche, F. Ellinger, and F. Jülicher (unpublished).

[33] R. Best, Phase-Locked Loops (McGraw-Hill, New York, 2003).

[34] L. Wetzel, D. J. Jörg, A. Pollakis, W. Rave, G. Fettweis, and F. Jülicher, PLoS ONE 12, e0171590 (2017).

[35] M. G. Earl and S. H. Strogatz, Phys. Rev. E 67, 036204 (2003).

[36] F. Dai, S. Zhou, T. Peron, W. Lin, and P. Ji, Phys. Rev. E 98 , 052218 (2018).

[37] S. Gerschgorin, Izv. Akad. Nauk. UdSSR Otd. Fiz.-Mat. Nauk 7, 749 (1931).

[38] L. S. Pontryagin, Am. Math. Soc. Transl. Series 2 1, 95 (1955).

[39] L. Wetzel, D. Platz, and A. Pollakis, github.com/cuichi23/ sim_pll_networks (2021); (a) set the system parameters in the dictionaries in the script; (b) start simulation with python $\langle$ scriptname $\rangle$.py.

[40] N. Punetha and L. Wetzel, arXiv:1908.11085.

[41] G. Giacomelli and A. Politi, Phys. Rev. Lett. 76, 2686 (1996).

[42] M. Wolfrum and S. Yanchuk, Phys. Rev. Lett. 96, 220201 (2006).

[43] M. Lichtner, M. Wolfrum, and S. Yanchuk, SIAM J. Math. Anal. 43, 788 (2011).

[44] M. Wolfrum, S. Yanchuk, P. Hövel, and E. Schöll, Eur. Phys. J.: Spec. Top. 191, 91 (2011).

[45] S. Ruschel and S. Yanchuk, Discrete Contin. Dyn. Syst.-S 14, 151 (2021).

[46] S. Goldman, Phase-Locked Loop Engineering Handbook for Integrated Circuits (Artech, London, UK, 2007).

[47] C. Hoyer, D. Prousalis, L. Wetzel, R. Riaz, J. Wagner, F. Jülicher, and F. Ellinger, in Proceedings of the 2021 IEEE International Symposium on Circuits and Systems (ISCAS) (IEEE, Daegu, Korea, 2021), pp. 1-5.

[48] E. Toscano and L. Lo Bello, in Proceedings of the 2008 IEEE 
International Workshop on Factory Communication Systems (IEEE, Dresden, Germany, 2008), pp. 139-148.

[49] A. Solovev and B. M. Friedrich, New Journal of Physics 24 013015 (2022).

[50] M. K. S. Yeung and S. H. Strogatz, Phys. Rev. Lett. 82, 648 (1999).

[51] J. A. Acebrón, L. L. Bonilla, and R. Spigler, Phys. Rev. E 62, 3437 (2000).

[52] L. Wetzel, F. Jülicher, D. Jörg, G. Fettweis, W. Rave, and A. Pollakis, Self-synchronizable network, European patent 2957982, 2014.

[53] P. Loschmidt, G. Gaderer, N. Simanic, A. Hussain, and P. Moreira, in Proceedings of the 2009 IEEE SENSORS (IEEE, Christchurch, New Zealand, 2009), pp. 781-786.
[54] M. Punturo, M. Abernathy, F. Acernese, B. Allen, N. Andersson, K. Arun, F. Barone, B. Barr, M. Barsuglia, M. Beker et al., Class. Quantum Grav. 27, 084007 (2010).

[55] H. Schuh and D. Behrend, J. Geodyn. 61, 68 (2012).

[56] A. Freeman, B. K. Malphrus, and R. Staehle, in Cubesat Handbook, edited by C. Cappelletti, S. Battistini, and B. K. Malphrus (Academic Press, San Diego, 2021), pp. 67-83.

[57] S. Ruan and J. Wei, Dyn. Contin. Discrete Impulsive Syst. B 10, 863 (2003).

[58] M. Komarov, S. Gupta, and A. Pikovsky, Europhys. Lett. 106, 40003 (2014).

[59] D. Métivier, L. Wetzel, and S. Gupta, Phys. Rev. Res. 2, 023183 (2020). 